\title{
Best International Practices of Combating Terrorism and Organised Crime by Special Units and Law Enforcement Agencies
}

\section{Meilleures Pratiques Internationales De Lutte Contre Le Terrorisme Et La Criminalite Organisee Par Des Unites Speciales Et Des Services De Detection Et De Repression}

\author{
Pavlovska Nataliia \\ PhD of Juridical Sciences, Associate Professor, Professor of Department of \\ Civil Law and Process of the National Academy of Internal Affairs, Kiev, Ukraine \\ ORCID ID 0000-0003-3311-0364 wwwpav@gmail.com \\ Kulyk Maryna \\ PhD of Juridical Sciences, Associate Professor of Criminal Produce of the \\ National Academy of Internal Affairs, Kyiv, Ukraine \\ ORCID ID 0000-0003-1373-6749 coolss777@ukr.net

\section{Tereshchenko Yuliia} \\ PhD of Juridical Sciences, Professor of the Department of Criminal Procedure of \\ the National Academy of Internal Affairs, Kyiv, Ukraine \\ ORCID ID 0000-0002-5353-0887 vladysikter@ukr.net

\section{Strilets Halyna} \\ PhD of Judicial Sciences, Associate Professor of the Departament of Law of \\ Prydunai Branch of Private Jointstock Company «Higher Educational Institution \\ of Interregional Academy of Personnel Management», Izmail, Ukraine \\ ORCID ID 0000-0002-1067-0820 galinastrelets2018@gmail.com
}

\section{Symchuk Anatolii}

Senior Teacher at the Department of Criminal Procedure of the National Academy Internal Affairs, Kyiv, Ukraine ORCID ID 0000000286638210 symchukas@gmail.com

\footnotetext{
Abstract

Therefore, this unit as a component of the gendarmerie is built on the principle of a military unit. The gendarmerie, one of the few state institutions in France, has been in existence for over 200 years and has a status as
} 
a significant component of the country's armed forces and is an extremely important part of the police system. The gendarmerie is subordinated to the Ministry of Defense (on the authority of the Main Directorate), and on the ground - to the command of military districts. At the same time, the gendarmerie is at the operational disposal of the Ministry of Internal Affairs and the Ministry of Justice. Significant autonomy within the Armed Forces allows the gendarmerie to combine military functions with purely police and administrative ones. The difference between police and gendarmerie is that the police are civilian civil servants. They can wear civilian clothes and trade union and political freedoms. Gendarmes also have the status of servicemen and military ranks, always in uniform, not entitled to strike and are responsible for violations in accordance with military charters - from guardians to dismissal from service (for example, for the use of alcohol "in the performance of official duties" the gendarme is threatened arrest for up to 30 days).

The need for the creation of the Austrian Special Forces was conditioned by the urgency of taking measures to ensure the safety of the flow of emigrants of Jewish nationality from the former USSR since in autumn 1973 against them was committed serious terrorist act. Special unit "Cobra" enters the warehouse of the Ministry of Internal Affairs and has got a double subordination: through direct combat engagement to the head of public safety, and in relation to personnel issues and logistics - the central command of the gendarmerie of the Austrian Ministry of Internal Affairs.

Among the well-known British Special Political Service (Special Air Service, or SAS) is probably the best counterterrorism unit. Its component - Special Projects (SP) team - the main anti-terrorist squad. The Special Air Service and its Counter Revolutionary Warfare Squadron (CRW) unit, the Antirevolutionary Military Squadron, were founded in 1942. The feature of training SAS servicemen is to teach each soldier to possess all methods and means of combating terrorism. To achieve this, SAS trains all of its squadron through training cycles. Acquired skills are improved later in the SP-team's combat duties. The main thing in the work is the maximum approximation of training sessions to a real combat situation in the conduct of operations on the release of hostages, in the role of which are civilians. Anti-terrorist training of SAS and the development of practical measures for the release of hostages is facilitated by the fact that high-ranking members of the British Government, including the Prime Minister, are personally involved in it.

Key words: international experience, organized crime, terrorism, gendarmerie, special units, crimes, armed units, military units, legal community but also sociology, temporary restriction of civil rights of citizens, civil legal relations.

Mots clés: expérience internationale, crime organisé, terrorisme, gendarmerie, unités spéciales, crimes, unités armées, unités militaires, communauté juridique mais aussi sociologie, restriction temporaire des droits civils des citoyens, relations juridiques civiles.

Un processus de réformation d'un système juridique de l'Ukraine provoque une attention de la direction MVS à l'expérience que accumulèrent des formations policières des pays étrangers, surtout nos voisins de l'Europe. Profitable peut être "une maquette française" colorite et originale, qui prévoit la présence dans l'Etat de deux policières charpentes - la police et national et la gendarmerie nationale. Gendarmerie - un des seuls instituts étatiques en France - existe plus de 200 ans, a un statut d'un composant essentiel des forces d'armées pondérables de pays et est une partie extraordinaire d'un système policier. NG est subordonné au ministère de la défense (à un droit d'une administrationprincipale), et sur place - au commandement des arrondissements armés. Simultané la gendarmerie se trouve dans la disposition du ministère de justice. Une autonomie considérable dans un cadre des forces armées permet au NG cumuler des fonctions armées avec les policiers et administratif. Dans notre pays après une révolution le mot "un gendarme" contracta une négation. Q propos une figure d'un gendarme dans une bleue uniforme et traditionnel képi est un des symbolesde la France (comme un gardien anglais au carême de la Grande Bretaigne). Le mot "gendarmerie" dans un mandat littéral signifie "un gens de l'arme" (de la sorte on appelèrent des chevaliers compagnes choisies). Une importance actuelle celui-ci a eut en 1791, 
lorsque la gandarmerie fut constitué à une façon d'un aspect des forces policières. Actuellement une attribution de $\mathrm{NJ}$ se épand à peu près sur $95 \%$ de territoire du pays, y compris les villes avec la une population moins de 10 milliers. Même dans des grandes cités la population a un droit d'élire, à qui s'adresser pour l'aide: au commissariat où au gendarme brigade. Dans les deux formations il y a des personnes qui ont la mandats policiers, puor faire les enquêtes criminels par un procureur peut être affecté un policier, et un officier de NG, et fréquemment - des délégués de deux formations. Bien clairement est que le facteur d'une compétition améliore un accomplissement des interrogations dans les intérêts de la justice. Cependant l'Etat a une grande importance d'une collaboration de deux charpentes - à celui-ci contribuent des circulaires communes MVS et le Ministère de la défense, la présence des officiers aînés des délégués de NG dans des meilleurs états-majors policiers, des particularités de l'organisation de la structure de la gendarmerie dont on voit le régime étatique et un décroisement de la France administratif à un degré national et régional $[1,16]$.

Une différence entre la police et NG se conclut de celui, que des policiers sont des étatiques employés civiques. Ils peuvent arborer une affaire civique et bénéficient des libertés syndicales et politiques. Les gendarmes ont un statut des militaires et une guerre dignité, toujours arborent une facture, non pas le droit à une grève et assument une responsabilité d'une contravention dont des guerres chartes de prison contre une délivrance du service (par exemple, sous un emploi d'alcool "sous un accomplissement" un gendarme menace une arrestation de délai 30 jours). Une administration NG principal occupe le directeur général - un agresseur civique.A lui et au Ministre de la défense s'inclinent un groupe spécial d'une sécurité, un groupe antiterroristique et des chefs des arrondissements NG. Un adjoint du directeur général est un inspecteur général - un général, dont coordonne une activité de NG avec le Ministère de la défense, contrôle tous les besognes des subdivisions et 12 institutions, actualise une association entre le ministre de la défense et la police nationale. Les interrogations législatifs de la national gendarmerie sont pareilles: un maintien de la discipline publice, une prophylaxie des crimes, assurer la sécurité d'un cheminement routier; un accomplissement des commissions des constitutions judiciaires, faire des enquêtes, une collection des démonstrations et une fixation des coupables avant le commencement d'une conséquence; une escorte de la communication, des objets étatiques importants, assurer la discipline dans des forces armées (en particulier, une lutte contre des déserteurs, un accompagnement des guerres colonnes, une admission et un démarrage des appelés); une prestation d'un aide à la population pendant les situations extraordinaires (dans une interaction avec des forces de la défense civique); un accomplissement des interrogations dans les intérêts des autres ministères, par exemple, selon une interrogation du Ministère des finances une collection des amendes, des créances, un contrôle des brevets commerciaux [5]. 
La gendarmerie nationale se compose d'un département (territorial) de la gendarmerie, de la gendarmerie mobile, d'une garde républicaine et des formations spéciales. Dans chaque arrondissement militaire de la France il y a un commandement NG, dont subordonné 3-4 légions (en fonction des districts économiques). Légion - est un accouplement avec le brigadier général, dont d'un aide d'un moyen étatmajor actualise un commandement des subdivisions NG dans un district. La légion se compose de 3-5 groupes gendarmes (au-delà de la quantité des départements). Un groupe - est une fraction qui est menée par un colonel où par un lieutenant-colonel - se compose de 3-4 compagnies qui sont menées par le commandant où le capitaine. Une compagnie en fonction de la composition et de la population, se compose de 7 jusqu'aux 20 brigades, et aussi un groupe d'administration, d'un compartiment de conséquence et un peloton d'aide auxiliaire qui est mené par un sous-officier [6].

Des brigades gendarmes (primaire subdivision de NG), les chefs sont des sous-officiers, s'installent dans une majorité des cantones de la France et sont de 6 aux 55 personnes. En générale, dans un pays campagnard à un millier d'habitants compte un gendarme seul. Dans des districts municipaux, où aussi exerce la police, à un gendarme seul compte cinq milliers d'habitants. A un armement département de la gendarmerie se trouve un chasseur d'arme, des autos et des camions, des transmissions aisées (y compris - électroniques) [7].

Une réserve de gouvernement puissante dans le cas des circonstances extraordinaires et pour une conduite de défense territoriale est 17 milles de la gendarmerie mobiles élevé dans 9 légions. Dans leur composition il y a 26 groupes de 130 escadrons: 23 groupes hybrides de 4-6 escadrons; une armée des chars et BTR aisés (huitescadrons); un groupe d'une escorte des objets nucléaires (trois escadrons); un groupe spécial, dont une composition des escadrons parachutes. Des escadrons (130-140 personnes) sont menés par un officier comme un capitaine. Une destination particulière dans une composition de NG emploie une garde républicaine, dont assure une sécurité du parlement et d'autres constitutions étatiques de la France, des résidences d'une direction de l'Etat, accentue des gardes honoraires et l'escorte pour des cérémonies officielles. Dans une composition des 12 compagnies et 5 escadrons d'une garde - des subdivisions des cavaleries, dont non seulement escortent un président et des chefs des Etats étrangers, mais assument un service d'une patrouille dans un borne de Paris grand. Des légions aussi engagent des formations spéciales: des routiers (desservent des chemins dans des campagnes); la gendarmerie marine (assure une sécurité des objets VMS et la gendarmerie des eaux territoriales); une compagnie de la sécurité marine sur la Ronne; des subdivisions des monts (des brigades, des patrouilles et des sauvent pelotons, y cimpris le seul dans l'Europe le peloton parachute de mont); la gendarmerie aériene, dont gardedes objets de VPS; la gendarmerie du transport aérien (une sécurité dans des aéroports civiques); la gendarmerie d'armement (garde des entreprises militaires et des dépots); la gendarmerie sous-marin, dont deux légions gardent un dispositif dans des colonies françaises sur les îles de Antiles et dans une 
Polynésie; et aussi - une auxiliaire gebdarmerie, dont les subdivisions se composent des militaires d'un délai service. Les gendarmes délais desservent pendant un an exceptionnellement comme des chauffeurs, des attachent et celui pareil, parce que les attirer à une escorte d'un dispositif public est défendu [8].

D'ailleurs, des officiers et des sous-officiers agencent plus de $80 \%$ des intérêts effectifs de NG, dont avaient au début de 1997 plus de 93 milliers de personnes sous celui, que la population de la France agencent lus de 57 millions. Dans un cas de guerre le nombre de la gendarmerie à une addition d'une mobilisation des reservistes peut promptement augmenter vite en deux fois. Dans un processus d'une réforme militaire, dont fait le président Jeac Shirak, la gebdarmerie Nationale dépassera au complectement selon un contrat. Aussi on doit agrandir un cercle des interrogations de NG selon une défense territoriale, une lutte contre le terrorisme et une contrebande de drogues. C'est pourquoi les étatsmajors de la gendarmerie jusqu'à 2002 dépasserons 97 milliers d'hommes [9].

Aussi faut noter, que les subdivisions gendarmes s'engagent à une composition de tous les contingents des forces français d'ONU et gardent des représentations diplomatiques de la Franceen étranger. Des français frugaux se portent contre des gendarmes mieux que contre des policiers, ils pensent que la gendarmerie est mieux disciplinée et travail mieux, est plus près à la population et moins de négation. L'episode avec le célèbre Lois Défunes nous montre l'opinion des gens: dans des films des héros-gendarmes sont comiques en leur consérvatisme et subordination mais sont bien disciplinés et font leur mition comme il faut. Ils montrent l'inventivité et à la fin gagnent tous les ennemis [10].

Dans une ville touristique tristing qui est située á $30 \mathrm{~km}$, au Sud de la capitale d'Autriche sur la porte d'un vieux Château il y a une armes sur laquelle on voit une Kobra qui est tournée autour d'Une épée avec deux grenades dans sa base. Ici setroure une subdivision spécialle du Ministére des Affaires intérieures d'Autriche qui s'occupe du terrorisme et a le nom «Kobra» [2].

Une nécessité d'une naîssance du speznaz d'Autriche était important pour la sécurité des émigrants européens du ex l'URSS après que pendant le printemps de 1973 il y eut une action terroristique contre eux. Une subdivision Kobra fait partie du Ministère des Affaires intérìeures et a deux Chef: de la ligue de combativité c'est le supérieure des affaires de la sécurité sociale et c'est aussi en ce qui concerne les cadres et les materieux et la téchnique - c'est la jandarmerie centrale MVS d'Autriche. C'est pourquoi cette subdivision (fait partie de la police) est comme une partie militaire a une structure: état - major (40 personnes) et 5 référateurs (secteur) - de la tachtique et des affaires de la combativité, d'organi sation, d'occupation, d'enseignement, de la téchnique, du materìeux. Le premier rapport ce sont les 8 groupes combatives (20 personnes dans chaque un). La subdivision d'enseignement est ailleur. Au total le nombre du personnel au «Kobra» est 150 personnes (ici: 13 ce sont les officiers). Les tâches principales de la speznaz sont: l'organisation de la préparation du personnel pour le combat des violateurs d'Autriche qui sont contre les loies des actions terroristiques et autres choses d'extrime; une lutte contre les terroristes, la 
délibération des prisoniers; une sécurité des passagers pendant les voyages en air ou par les trains; la défense des personnes conues et des chefs d'Etats (qui sont toujours au risque d'être tué); une sécurité des visites des chefs d'Etats et des supérieurs étrangers; la défense d'un aéroport international Shvehat (avec un groupe spécial «jouravel» (sigogne)); une sécurite des conférences internationales, des samposiums; le contrôle des visites á Vienne des émigrants, leur sécurité. Alors, au total les tâches de «Kobra»sont les mêmes que dans autres Etats en ce qui concerne les subdivisions en lutte contre le terrorisme. Les conditions pour entrer dans la subdivision d'Autriche sont trés intéressants. Pour entrer en «Kobra» il faut bien passer les examens. Pour le service on prend les hommes (du 20 ans - à 45 ans) qui on terminé deux ans de l'école de la jandarmerie et ont de la pratiques du service à la police. Ils ne doivent pas avoir une famille. Ce n'est pas parce que c'est un service spécial (ils vivent dans les kazarmes), mais c'est aussi grace aux risques qu'ils ont pour la vie. Les personnes qui veulent faore leur service militaire dans ces subdivisions doivent écrire le rapport et marquer ici le nom de leur propre chef et aprè cela passer les examens d'entrés qui ont lieu au mois de mars et aoùt. Le service militaire a des conditions très difficiles. Les personnes qui veulent y entrer passent les examens - tests qui ont une vérifications d'intellect et les épreuves physiques et phsycologiques. Les candidats (impétrans) passent les examens des disciplines de la spécialité: conduire la voiture et tirer des armes différentes [3].

Pour augmenter l'exigence et faire les conditions qui conviennent pour la concurence entre les combateurs on fait des tests et des examens dans la subdivision tout les trois mois qui montrent la préparation des spéznazients pour faire les tâches. Et c'est pour ça que tout les trois mois les 10 personnes qui ont les résultats les plus mauvais sur la liste doivent s'en aller. On les remplace par les autres qui ont bien passer ses examens et la préparation dans le rapport d'enseignement. Les personnes qui ont connu l'échec retournent à la jandarmerie. Mais il faut dire que après avoir passer le service dans la subdivision du speznaz ces gens reçoivent l'expérience qui leur aide dans le travail. A partir de ce temps là ils sont des modèles pour les collègues. On ne peut pas dire que ce sont des vrais échecians parce que d'après les règles 10 personnes doivent partir $\mathrm{du}$ «Kobra» toujours obligatoirement même si ils sont aussi forts que les autres. Ces gens sont les derniers des meilleurs et ça ne veut pas dire qu'ils sont les pirs. Ce mécanisme du regénération permanente fait que la combativité est toujours haute. Les personnes qui rassent les examens signent un contrat pour 2,5 ans et passent aux troisième rapport où ils suivent des courts spécieux (la préparation). L'enseignement est le processus de différention et contient la préparation générale et les courts spécieux où on prépare des nageurs-sous-mariniers, des savants d'explosition, des radistes et autres spécialistes. «Kobra» est une subdivision qui est toujours prête pour la lutte c'est pourquoi l'organisation de la journée et du service est bien calculée. Le groupe anquiétant peut toujours arriver sur la place d'événement aux moments différents: 20 personnes du premier rapport; 10 spécialistes d'éxplosition; des radistes; 2 officiers d'administration «Kobra». A part, le groupe a une voiture 
d'urgence (d'aide médicale) avec les médecins. La partie principale de la subdivision a les courts d'après le plan de la préparation militaire. Il est intéressant que en s'occupant de l'état phsycologique du personnel on donne à chaque homme chaque mois le repos de 4 jours. C'est une pratique positive quand ils passent les difficultés à «Kobra». L'exigence principale pour le groupe auquiétant-être à l'heure pendant l'événement. Par exemple, pour aller à l'aéroport Shvehat, qui est situé à $30 \mathrm{~km}$ des kazarms de «Kobra» le groupe doit être sur place après 20 minutes du signal. Donc, le trait commun pour le GSG-9 allemand et pour «Kobra»d'Autriche c'est la mobilisation parce qu'ils ont un bon mécanisme de travail [4].

Pour les membres du speznaz d'Autriche l'état physique est très important. Ici les exercices de combat corps à corps sont importants mait aussi la vitesse de la réaction, l'endurance, la coordination des mouvements pendant la nuit. Pour se préparer et être en bon état physique on leur donne $20 \%$ du temps du processus d'enseignement. 24 heures sur semaine c'est le temps pour les courts de tir avec les armes différentes y compris la production étrangère qui est utilisée par les terroristes. Sur un poligon spécial on fait des situations différentes où peuvent se trouver les travailleurs du «Kobra». Ils tirent de la position «par terre», debout (toute de suite après $5 \mathrm{~km}$ de course), pendant le mouvement, du hélycoptère qui est en air, de la voiture qui passe à une grande vitesse. Pour faire les recherches-aides à «Kobra» on prépare les groupes des nageurs-sous-mariniers. La base de l'air-descent préparation c'est l'enseignement des sauts avec les parachutes de l'avion et des hélycoptères (le jour et la nuit). Il y a un court spécial des tireurs de précision [11].

Chaque membre de «Kobra» a le pistolet MP-73, automate «Shtailler 77», des grenades avec le gaz, l'arme blanche, les matérieux pour voir la nuit, la radiostation portative et le bronigilet de la production anglaise (10 kilot). A partir de 1981 dans chaque territoire d'Autriche on a commencé à créer les subdivisions comme les speznaz mais ils ont des traits différents et non pas la téchnique comme «Kobra» et non pas le si grand nombre des membres et de la préparation du personnel. Le speznaz a beaucoup gagné en lutte contre le terrorisme, a eut du succès. Sur le drapeau de la «Kobra» on voit: «Le terrorisme n'a aucune chance». Ce slogan n'est pas qu'une phrase vide. Le dernier temps le pays n'a pas su d'actions de terrorisme par des autrichuens. C'est le travail des spécialistes du «Kobra». Le role principal ici a le terrorisme international qui est répendu en Autriche aussi. En 1975 ils ont fait une attaque pendant la conférence OPEK. En 1981 - la série des attaque terroristiques contre les virments et les immigrants turcs. En juillet 1989 des terroristes ont tué le secretaire général du parti Démocratique d'Iran Kourdistan et son adjoint.

Tout cela veut dire qu'il y a une nécessité d'une lutte importante avec le terrorisme sur le territoire d'Autriche. C'est porquoi la police d'Etat a creé un groupe spécial qui s'occupe du dévélopementdes liaisons avec d'autres pays et fait des échanges d'information. «Kobra» reçoi aussi évidement 
l'information. En ce qui concerne le travail du speznaz avec les subdivisions des autres pays dans le domaine du terrorisme il faut dire qu'en Autriche pour passer les courts d'après une accorde arrivent toujours des spécialistes de l'Italie, FRN, de Bélgique, Chine, France, Suisse, USA, Espagne, Tunisie, Pérou, Malta. Comme ça on peut voir les niveaux des spécialistes. Dès 1980 à Tristing on a toujours (tout les deux ans) des réunions des supérieurs des subdivisions en lutte contre le terrorisme des pays différents: Autriche, USA, FRN, Grande Bretaine, Italie, France, Suisse [12].

Parmi tous les célèbres un spécial aérien service (Special Air Service, ou SAS) britannique, probablement doit etre la mailleure subdivision spéciale selon une lutte contre le terrorisme. Ses éléments sont - Special Projects (SP) team (Un commandement des projets spéciaux) - un peloton essentiel contre le terrorisme. Un aérien service spécial et sa subdivision Counter Revolutionary Warfare Squadron (CRW). Un escadron antirévolutinaire militaire furent basé en 1942. Leur état-major situé au Héréforde (Grande-Bretagne). SP commandement se compose à peu près de 80 personnes, dont sont décroisés en 4 pelotons seize personnes dans chacun. Pendant le cycle des cours SP collabore avec autre escadrons. Et lorsqu'un acte de terroriste se produit, un peloton combatif se partage en un aperçu,des tireurs de précision et un groupe d'enlèvement. Tous les SAS-escadrons ont un devoir d'assumer un gardes combatives à travers d'une section antirévolutionaire militaire. Les cycles d'entraînements dans CRW-escadrons communément durent un croissants six mois. Vu le terrorisme, à l'exception des groupes spéciaux à propos de l'approvisionnements physique des cas, existe un département d'une exploration des opérations, dont non seulement conclut l'interrogations d'une lutte tactiques contre leterrorisme, mais aussi exploite un aménagement unique pour SP-commandement. Dans particulier, des grenades, des degrés spécialisés pour faire les opérations spéciales dans les trains,les avions les aménagement nocturne. Une particularité d'une formation des militaires SAS se conclut de ce que chaque combattant doit savoir tous les méthodes pour une lutte entières contre le terrorisme. Pour avoir le résultat SAS enseigne tous ses escadrons avec l'aide des cycles d'entraînements. C'est pourquoi les travailleur SAS considèrent comme les spécialistes avec un bon niveau selon une lutte contre le terrorisme: premièrement tous eux savent parfaitements utiliser l'arme mais aussi les méthotes de combat; deuxièmement, assurent les cycles d'entraînements administrés [13].

Organisationnel SAS a deux commandements: les Rouges et les Bleues dont au total se compose de 65 personnes Dans chaque groupe il y a les tireurs de précision et des experts. Un art de SAS, d'une façon comme allemand GSG-9 et autrichien "Kobra", se conclut d'une application habile de l'arme. Pour avoir du succès en tir fut expressément exploité un cap d'étude essentiel, durant six semaines. Pendant ce cycle les soldats doivent faire à peu près 2000 exercices. Les habitudes contractées s'épurent par la suite à l'heure des gardes combatives SP-commandements. Le principal dans le travail c'est une approche maximale des entraînements pour une ambiance combative pendant les opérations à 
propos de délivrance, dont le rôle avancent les citoyens civiques. L'enseignement antiterroristique SAS et la pratique selon une délivrance des otages se facilite d'une façon qu'ici individuelement acceptent une participation les membres du gouvernement y compris le premier-ministre. Pour assurer une mobilité de SAS utilisent (dans un cas de besoin) des forces aériennes royales C-130, dont toujours demeurent dans une réserve, parce qu'en n'importe quel époque leurs bienfaits peuvent servire pour le service.

Disposé de n'importe quel groupe special selon une lutte contre le terrorisme se détermine non seulement d'une qualité des cycles d'entraînements et une quantité des habitudes selon une délivrance des otages, capturé dans un avion, en autos, mais aussi une participation pragmatique des combattants immédiat dans une liquidation des terroristes. SAS accepta une participation dans des opérations antiterroristiques. Surtout dans l'Irlande arctique et empocha des rappels approbateurs de la part du département britannique [14].

Intéressant ce que, nombreuses formations dans le monde entier, en particulier nouvellesZélandiens et australiens, aussi utilisent le nom SAS. Une longue époque continuent des débats à propos d'utilisation d'un légendaire spécial groupe (Special Boat Service, ou SBS) britannique pour une lutte contre le terrorisme. Donc il en existent des idées différentes, mais des experts divers pensent, que SBS non fut déployé, si n'avait pas eu lieue une échelle incident terroristique, dont SAS décela individuelement. SAS et SBS, d'une façon célèbre, agissent ensemble pendant un dépistage des agencements explosifs. Justement à cause de la force pendant la formation des combattants SAS ce service se porte assuré sur le sol, et dans l'air et sur la mer.ça montre encore un coup positive de l'enseignement de chaque combattant SAS. Théoriquement on peut décrire la situation, lorsque les actes terroristiques peuvent se produirent dans plusieurs places en meme temps dans un pays. Un exemple pareil put être un enlèvement simultané de plusieurs plates-formes pétrolières dans le Mer arctique. Bien sur que, c'est une fantaisie, mais même un cas pareil est prévu par les spécialistes de SAS et SBS. Donc, si un cas pareil se produit, des services spéciaux sont prêt à réagir adéquatement. Il fautdire qu'entre SAS et SBS existe un grand degré de collaboration. Donc SAS est un mailleur partenaire dans un échange de l'information à propos d'une lutte contre le terrorisme. Des combattants de ce service administré déléguèrent leur expérience aux collègues de The United States`Delta Force, the FBI`s Hostage Rescue Team (HRT), France`s GIGN, Germany`s GSG-9, Spain`s GEO, the Royal Dutch Marines et SAS-un groupes et la Nouvelle-Zélande. Cet échange de l'information eut un grand effet et sensiblement contribua une augmentation d'habitudes dans une lutte contre le terrorisme dans le monde entier. Des combattants de SAS avancèrent d'une façon des conseileurs pendant les études des subdivision selon une lutte contre le terrorisme dans les pays différents. Mérite une attention le fait qu'avant à une époque déterminé exista une méfiance de SAS contre le service spécial israélien MOSSAD. À cause de cela SAS abandonna accepter une participation dans n' importe quel études ni programmes communes d'un 
échange d'information. Cependant le dernier temps les liens entre ces deux groupes spécieux se stabilisèrent, qui fait éventuelement la possibilité des opérations antiterrorustique communes. Des combattants SAS acceptent non seulement la participation immédiate dans l'arrêt des actes terroristiques, mais accordent des consultations avec les services pareilles d'autres pays à propos de l'organisations des opérations spéciales. Comme ça, six combattants SAS, deux officiers et quatre officiers d'une composition aînée furent adressé à Lime dès décembre 1996 jusq'à avril 1997, pour aider au gouvernement pérouant de resoudre un problème d'un enlèvement un otages [15].

Dans un aboutissement de cette opération on a sauvé 71 persones de 72 otages. Une chance dans la lutte contre le terrorisme se conclut d'une collaboration des subdivisions du monde entier. Vu une expérience positive et des contresens des collègues, on peut conjurer des facteurs négatives, dont accompagnent les opérations spéciales, et aussi prendre un armement les meilleures méthodes de la lutte contre terrorisme.

\section{References}

1. Ukrai'na-Francija: dogovirni vzajemovidnosyny u gumanitarnij ta pravovij sferah. [Ukraine-France: contractual relations in the humanitarian and legal spheres]. URL: http://elar.naiau.kiev.ua/jspui/handle/123456789/5466

2. Mizhnarodnyj teroryzm: konsolidovanyj analiz zabezpechennja bezpeky [International terrorism: a consolidated security analysis]. URL: http://elar.naiau.kiev.ua/jspui/handle/123456789/5464

3. Antyterorystychnyj specnaz: syly, zasoby i tehnologii' protydii' teroryzmu, organizovanij zlochynnosti $i$ korupcii' (vitchyznjanyj i svitovyj dosvid psyhofiziologichnoi', profesijnoi' i special'noi' kryminalistychnoi' pidgotovky). [Anti-terrorist special forces: forces, means and technologies of combating terrorism, organized crime and corruption (domestic and world experience of psychophysiological, professional and special forensic training).] URL:

http://elar.naiau.kiev.ua/jspui/handle/123456789/5453

4. Antyterorystychni syly, zasoby, tehnologii' bezpeky: konceptual'ni osnovy zapobigannja ta protydii' teroryzmu. [Anti-terrorist forces, means, technologies of security: conceptual foundations of prevention and counteraction to terrorism.] URL: http://elar.naiau.kiev.ua/jspui/handle/123456789/5454

5. Lipkan V. A. Administratyvno-pravove reguljuvannja nacional'noi' bezpeky Ukrai'ny [Tekst] : Monografija /V. A. Lipkan. K. : Kyi'vs'kyj nac. un-t vnutr. sprav, 2008. 440 s. [Administrativelegal regulation of national security of Ukraine [Text]: Monograph / V. A. Lipkan. K.: Kiev NATION. UN-t inside. Affairs, 2008. 440 p.]

6. Lipkan V. A. Nacional'na bezpeka Ukrai'ny [Tekst] : normatyvno-pravovi aspekty zabezpechennja / V. A. Lipkan. K. : Tekst, 2003. 180 s. [National Security of Ukraine [Text]: Regulatory aspects of provision / V.A. Lipkan. K.: Text, 2003. 180 p.]

7. Lipkan V. A. Nacional'na bezpeka Ukrai'ny [Tekst] : navch. posib. / V. A. Lipkan. 2-e vyd. K. : KNT, 2009. 576 s. [National Security of Ukraine [Text]: Teach. manual. / V. A. Lipkan. 2nd species. K.: KNT, 2009. 576 p.]

8. Lipkan V. A. Teoretyko-metodologichni zasady upravlinnja u sferi nacional'noi' bezpeky Ukrai'ny [Tekst] : monografija /V. A. Lipkan. K., 2005. 350 s. [Theoretical and methodological principles 
of management in the sphere of national security of Ukraine [Text]: Monograph / V. A. Lipkan. K., 2005. $350 \mathrm{s.}]$

9. Lipkan V. A. Teorija nacional'noi' bezpeky [Tekst] : pidruchnyk /V. A. Lipkan. K. : KNT, 2009, 631 s [Theory of national security [Text]: Textbook / V. A. Lipkan. K.: KNT, 2009, 631 s].

10. Lipkan V. A. Nacional'na bezpeka Ukrai'ny: kryminal'no-pravova ohorona [Tekst] : navch. posib. / V. A. Lipkan, I. V. Diordica. K. : KNT, 2007, 292 s. [National Security of Ukraine: Criminal Protection [Text]: Teach. manual. / V.A. Lipkan, I.V. Dordica. K.: KNT, 2007. - 292]

11. Lipkan V. A. Nacional'na i mizhnarodna bezpeka [Tekst] : slovnyk / V. A. Lipkan, O. S. Lipkan. - 2-e vyd., pererob. ta dop.. K. : Tekst, 2008, 400 s. [National and International Security [Text]: Dictionary / V.A. Lipkan, O. S. Lipkan. - 2nd ed., processed and imp. K.: Text, 2008, 400 p.]

12. Lipkan V. A. Nacional'na i mizhnarodna bezpeka u vyznachennjah ta ponjattjah [Tekst] / V. A. Lipkan, O. S. Lipkan. K. : Tekst, 2008, $255 \mathrm{~s}$. [National and international security in definitions and concepts [Text] / V.A. Lipkan, O. S. Lipkan. K.: Text, 2008, 255 p.]

13. Criminal law and forensic classification of smooth-bore firearms. URL: https://doi.org/10.32370/IAJ.2079

14. Legal regulation of the firearms circulation in the countries of the former Warsaw treaty and the Soviet Union. URL: https://doi.org/10.25313/2520-2308-2019-2-4852

15. Aviation flights safety as an element of national security. URL: https://doi.org/10.25313/2617-572x-2019-1-4868

16. Bureau of alcohol, tobacco, firearms and explosives, (atf) USA: genesis. URL: https://doi.org/10.25313/2520-2294-2019-4-4855 